\title{
A Novel Mapping Technique for Ray Tracer to System-level Simulation
}

\author{
Muhammad Awais Khan ${ }^{\text {a,* }}$, Sherif Adeshina Busari ${ }^{a}$, Kazi Mohammed Saidul Huq ${ }^{a}$, \\ Shahid Mumtaz ${ }^{a}$, Saba Al-Rubaye ${ }^{b}$, Jonathan Rodriguez ${ }^{a, c}$, Anwer Al-Dulaimi ${ }^{d}$ \\ a Instituto de Telecomunicações, 3810-193 Aveiro, Portugal \\ ${ }^{\mathrm{b}}$ Autonomous and Cyberphysical Systems Center, Cranfield University, United Kingdom \\ c University of South Wales, Pontypridd, CF37 1DL, United \\ Kingdom ${ }^{\mathrm{d}}$ Center of Excellence, EXFO, Canada
}

\section{ABSTRACT}

Simulations have become remarkably useful in evaluating the performance of new techniques and algorithms in communication networks. This is due to its comparative cost, time and complexity advantage over the analytical and field trial approaches. For large-scale networks, system-level simulators (SLS) are used to assess the performance of the systems. The SLS typically employs statistical channel models to characterize the propagation environment. However, the communication channels can be more accurately modeled using the deterministic ray tracing tools, though at the cost of higher complexity. In this work, we present a novel framework for a hybrid system that integrates both the ray tracer and the SLS. In the hybrid system, the channel strength in terms of the signal-to-noise ratio (SNR) is fed from the ray tracer to the SLS which then uses the values for further tasks such as resource allocation and the consequent performance evaluation. Using metrics such as user throughput and spectral efficiency, our results show that the hybrid system predicts the system performance more accurately than the baseline SLS without ray tracing. The hybrid system will thus facilitate the accurate assessment of the performance of next-generation wireless systems.

\section{Introduction}

The aim of the fifth generation (5G) technology is to satisfy the explosive demand for higher capacity, massive connectivity, ultra-low latency and ultra-reliable communications so as to serve a variety of new use cases whilst supporting the legacy services and applications [1]. The new $5 G$ use cases will find application in autonomous vehicles, smart healthcare systems, emergency services, smart cities, intelligent industrial automation, virtual and augmented reality, among others [2]. To realize the $5 \mathrm{G}$ goals, some of the key innovative technologies that are being explored include ultra-dense small cell networks, massive multiple-input-multiple-output (MIMO), millimeterwave (mmWave) and terahertz ( $\mathrm{THz}$ ) band communication, softwaredefined network-ing, network functions virtualization, network slicing, etc. In both the industry and academia, several protocols, techniques and algorithms are being proposed with performance evaluation being undertaken to assess their potentials and capabilities.

The three main approaches employed in evaluating the perfor-mance of any new communication paradigm are numerical simulations, mathematical analyses and field trials. Though analytically tractable, mathematical methods (e.g., stochastic geometry tools) are often con-strained with simplifying assumptions that potentially limit their use in modeling largescale, highly-complex and dynamic networks. Realistic performance can be measured in live operating environments. However, the economic and operational requirement are costly, unsuitable and practically infeasible for the early design and development stages. Hence, simulations have become remarkably important tools, since the past few decades for the assessment of networks' performance due to obvious cost and implementation advantages. Simulations can provide the verification and validation of the key characteristics and behaviors of the overall system [3,4].

Depending on the performance metrics under investigation, simula-tors can be categorized into three: Link Level Simulator (LLS), System Level Simulator (SLS) and Network Level Simulator (NLS). While the LLS examines detailed, bit-level physical (PHY) layer functionalities of a single link, the SLS evaluates performance of links involving many base stations (BSs) and user equipment (UEs) at medium access control (MAC) layer, with the PHY abstracted usually through look-up tables. SLS focuses on the radio access network/air interface and facilitates analyses on resource allocation, capacity, coverage, spectral and energy efficiencies, among others. The performance of protocols across all lay-ers of the network, including control signaling and backhaul/fronthaul issues are evaluated with NLS using metrics such as latency, packet loss, etc. Besides metric-based classification, simulators can also be grouped based on radio access technologies supported (cellular, vehicular, WiFi etc.), coding language (MATLAB, python, $\mathrm{C}++_{+}$, etc.), licensing option

Corresponding author.

E-mail addresses: jadoon.awais@av.it.pt (M.A. Khan), sherifbusari@av.it.pt (S.A. Busari), kazi.saidul@av.it.pt (K.M. Saidul Huq), smumtaz@av.it.pt (S. Mumtaz), s.alrubaye@cranfield.ac.uk (S. Al-Rubaye), jonathan@av.it.pt, jonathan.rodriguez@southwales.ac.uk (J. Rodriguez), anwer.aldulaimi@ieee.org (A. Al-Dulaimi).

https://doi.org/10.1016/j.comcom.2019.11.039

Received 11 September 2019; Received in revised form 21 October 2019; Accepted 23 November 2019 Available online 2 December 2019 
(open source, proprietary, free of charge for academic use) or network scenario capabilities (LTE, 5G, B5G, etc.) [4].

In SLS, such as the Vienna SLS [3] widely used in the academic community, stochastic channel models are used in evaluating the signal to noise plus interference ratios (SINR) statistics using mathematical distributions. The results are then averaged over several channel re-alizations. It is instructive to note that these statistical values only provide a somewhat representative outlook of the propagation environ-ment. Using ray tracing engines, however, it is possible to characterize the communication channel in a deterministic manner thereby pro-viding accurate channel model and SINR values for the considered scenarios. The ray tracer uses detailed information of the environment to model the deterministic channel, including the terrain, building material, trees, foliage and other obstacles or blockages along the propagation path. To address the challenge resulting from non-realistic channel models in SLS, ray tracing tools [4] can be used to provide the deterministic and realistic results of the channel. The resulting signal-to-noise-ratio (SNR) values can then be fed to the SLS for further processing tasks such as resource allocation and performance evalua-tion. The ray tracer tool, on the one hand, provides the ray beam matrix information from real platforms, but this tool cannot simulate the entire network functionalities. On the other hand, the SLS can simulate more network functionalities for several BSs and UEs, but the values of the channel matrices are statistically estimated. The scientific community is, therefore, exploring different approaches, such as multilevel and hybrid approaches, to address the challenging tasks in complex wireless networks.

In this work, we employ a hybrid technique combining the features from both the ray tracer and the SLS. The system maps the realistic SINR values resulting from the realistic channel model from a ray tracer to the SLS values for further advanced computations. This hy-brid approach is very promising for accurate performance evaluation. However, a ray tracer generates a massive amount of data which consti-tutes a complexity challenge for the mapping process. Aggregation and replacement of the ray tracer values to the SLS is a complex task. To ad-dress this, we propose a low-complexity distance-based algorithm that maps the ray tracer values to the SLS based on the distance between the transmitter and the receiver. The intuition of the proposed method is to provide a balanced tradeoff between the computationally complex but very accurate ray tracer and the less computationally complex SLS with much lower granularity. It provides a better performance with lower complexity than the conventional baseline methods. To the best of the authors' knowledge, this work is the first to present such framework.

The remainder of this paper is organized as follows. An overview of the related works is provided in Section 2. The simulation framework is provided in Section 3. The proposed algorithm and the simulation scenarios are provided in Section 4. The results and discussions are pre-sented in Section 5 while the conclusions and future research directions follow in Section 6.

\section{State of the art}

Currently, many advanced simulators have been developed for cel-lular networks. To simulate the LTE system, [5] has designed a simu-lator to evaluate the performance of the downlink shared channel for LTE SISO and MIMO networks. In this work, the physical layer model is based on post-equalization SINR. Fading parameters are pre-calculated to reduce the computational complexity at runtime. Extended ver-sions of the systemlevel simulator are presented in [4]. In [6], a 5G simulation framework, called Network Simulator System (NSS), was developed in C++ to include advanced $5 G$ features. NSS provided a graphical interface to plan and establish a network in different densely populated regions. In [7], a SLS called WiSE was introduced for $5 \mathrm{G}$ mobile networks with the capabilities of channel modeling and radio resource allocation. Different memory management techniques were introduced and new features were implemented for the accurate simula-tions for different UE movements, their rotation and blockage behavior along with the underlying 3D beamforming architecture.

In [8], 5G and beyond scenarios are simulated using the Vienna 5G Link Level Simulator (LLS) and the SLS. The interaction of these simulators and their reliance on each other is verified and validated in the research. The LL simulation is input to the SL simulator and the overall abstraction and simplifying individual communication links can reduce the necessary complexity reduction and can make the process more flexible in terms of physical methods. For physical layer information between a transmitter and a UE, many ray tracing tools have been designed to get the real information of a beam array. In [9], indoor mmWave massive MIMO channel has been simulated with 1024 antenna elements. The simulations used ray-tracing simulator to calcu-late the path loss, shadow fading, root-mean-square delay spread, and coherence bandwidth.

A shoot and bounce reflected/transmitted ray-polygon method was used in [10] for better memory utilization with fast and accurate ray tracer values. A diffracted-reflected $(D-R)$ ray enhanced the accuracy of the system in a 3-D shooting and bouncing polygon. The experiments have simulated for the urban street canyon, indoor and tunnel propagation environments. In [11], a MAC layer protocol is proposed for indoor and outdoor hexagonal grid deployment scenarios with 3 different rings, such as indoor hotspot $(\mathrm{InH})$ and urban micro (UMi). The solution is proposed to reduce the interference in the inner ring with the spatial correlation of shadowing and line-of-sight (LOS) link. Results showed that modulation types and filter design could affect the performances due to out-of-band leakages of OFDM.

For personal radio frequency electromagnetic field (E-field) exposure in public transportation system was explored using 3D raylaunching (3D-RL) in [12]. E-field estimations were obtained from interior of urban buses for determining the exposure levels to the transceiver location within the bus by exploiting multipath propagation and shadowing characteristics. Results have shown that 3D-RL deterministic simulations offer better accuracy and computational cost than other solutions when the impacts of morphology and the topology of vehicles were considered.

In [13], a fixed and a mobile relay-based system level simulator is used to evaluate the OFDMA networks to enhance the spectral efficiency at the cell boundary. Different routing and medium access control decisions are evaluated in different multihop and relay interactions among the users. An efficient three-dimensional ray tracing (ETRT) method for the prediction of indoor radio propagation is proposed in [14] and compared with conventional shooting bouncing ray tracing (SBRT) method. Simulation results are tallied with actual measurement using path loss and received signal strength indication. For each multi-channel ray, the RT considered reflections and penetrations effects depending upon the uniform theory of diffraction and geometric optics. However, computational complexity is reduced by allowing more rays in observed zones but not in all directions.

Article Contribution: As the requirements of envisioned 5G, highly reliable communications are an essential ingredient for the next gen-eration of wireless networks. There has been a significant research coverage on characterization and improvement of individual mmWave features in the simulation. However, there is a lack of system-level performance with multiconnectivity in mmWave connections and not yet available in the current literature. The motivation for the design of the new framework is to harness the opportunities for accurate and realistic values provided by ray tracers (RTs) when simulating complex scenarios using system level simulators (SLSs). The hybrid system, thus, provides a balanced tradeoff as opposed to the computationally-complex RT-only simulations and the SLSs with slight reduction in accuracy due to higher map resolution or coarser granularity than RTs. The hybrid system facilitates a more accurate characterization of the system performance.

To observe the whole process of LTE in the real world is substantially expensive, that is why our focus is to simulate the whole process 
through these link and system level simulators. However, traditional method of using different link and system simulators in sequential processing is time-consuming, while our method to use the values from a real scenario in the SLS will reduce the time. Moreover, to validate the mathematical model describing the system due to the insufficient data is extremely expensive and this paper will use the simulation of the whole system by taking the real link layer values from ray tracer and using the overall characteristics from SLS. This proposed method will help better understanding of the whole system by testing the feasibility of simulating the values from ray tracer into SLS. It will also help compress time to observe the results with two different simulators in sequential order. In this paper, multiple performance metrics are used for analyzing the system which employs a systematic approach to problem-solving. Furthermore, it is possible to develop a well-designed and robust system through our proposed model and reduce the development time.

\section{Preliminaries}

System level simulations focus more on system-related issues such as scheduling, mobility handling or interference management. System level simulations are essential for the evaluation of the cellular com-munication networks, especially for having a detailed insight of the large-scale scenario and its performance. A real-world measurement approach becomes laborious and comes with a huge cost, so system level simulators are developed. On the other hand, link-level simula-tions provide the investigation of issues such as MIMO gains, adaptive modulation and coding feedback, and physical layer modeling of the system.

\subsection{Ray tracer}

In our paper, we used a 3D, polarimetric, and multithreading ray tracer called TruNET wireless. This simulator is based on a customdeveloped electromagnetic engine, developed by Sigint [15]. TruNET provides a deterministic radio simulation environment, which allows a realistic design of the wireless communication network. In TruNET wireless, the design of any wireless radio network can be done, such as cellular, wireless broadband, UWB, Wireless Sensor Networks (WSNs), Wi-Fi, etc. The standard output of the simulator provides magnitude and phase information, Power Delay Profiles (PDP), AoA, AoD infor-mation, SNR, and SINR. The simulator's core functionality is further ex-tended through several communication modules and connectors. Exam-ples of these modules include MIMO modules, higher layer simulation modules, localization modules that allow the creation of fingerprinting databases and their performance evaluation, jammer modules, geoloca-tion database Modules for TV White Spaces network design etc. TruNET has different connectors to interact with different simulating tools, such as NS2/NS3, MATLAB, Emulators, Communication Equipment (spectrum analyzer, network analyzer), and custom based modules as well.

The design of TruNET ray tracer has an intuitive CAD and antenna designer that helps in rapid design and configuration of the wireless networks under investigation. In our paper, we use a real-life ray beam information from this ray tracer tool and use them in a proven effective system level simulator. The ray tracer supports the estimation of multiple receiver area layers, for example, if the receiver area is constituted from 2 layers, then the lower layer is with a lower height and then the upper layer with more values. Fig. 1 (a) and 1 (b) explain the cells numbering with respect to a single layer and two layers, respectively. When a receiver area is deployed in a wireless ray tracer scenario, the lower left Cell ID always has the lowest ID number. For example, for a single layer, single receiver area, the lower Cell ID will have 0 . The next Cell ID sits on top of the previous Cell ID in the same column. Cell ID number increases until the end of the column (see Fig. 1(a) for Cell ID 3) and subsequently, Cell ID jumps to the bottom cell of the next column (Cell ID 4) and so on.

\subsection{System level simulator}

System level simulator (SLS) is a modeling simulator for system behavior at a higher level of abstraction with flexibility of support-ing multiple mmWave scenarios with realistic and synthetic layouts, antenna configurations, channel characteristics, and lower-layer 3GPP procedures. For a comprehensive simulator that can deal with complex, usable, scalable and open access, Vienna LTE-A downlink system level simulator is considered for our research work. The Vienna LTE-A system level simulator is an open source object-oriented simulator in MATLAB available under academic, non-commercial use license. It has features of resource allocation for femtocell networks, algorithms for self-optimizing networks, and machine-to-machine communications. It also provides advanced features like 3D channel modeling. Fig. 2 shows the link quality model and link performance model, and their relation to the whole LTE link to system model.

The capabilities and the limitations of ray tracer and SLS are mentioned in Table 1.

\subsection{Performance evaluation features}

Different parameters of the whole system were considered for simu-lation, such as user deployment, network deployment, mobility of users and blockers, user traffic demands, resource allocations, and blockage modeling.

User Deployment: The area is divided into a $600 \times 600$-meters zone. The area is further divided in resolution of $1 \times 1$-meter value. The users in a certain zone are divided randomly, but these values can be set appropriate to represent a real scenario.

Network Deployment: A single AP is considered in our scenario in principle at a location of $(0,0)$ coordinates. This AP is assumed as static but can be characterized as mobile AP under certain conditions. And $X$ number of users are deployed around the AP, e.g., vehicles, IoT devices, drones, etc.

Mobility of Users and Blockers: In our scenario, we have modeled all the users as mobile around the AP. Different mobility speeds are considered to verify the correctness of our system.

User Traffic Demands: The number of session types are designed to have a real traffic demand and service time in the network. Resource Allocation: The system resources are allocated with a typical session management, where new requests from users are only accepted if there are resources to serve them.

Blockage modeling: The blockage of radio propagation could be anything with a certain height and diameter, such as human, trees, buildings, etc.

It is important to investigate the above-mentioned significant factors to integrate the mmWave communications technology in $5 \mathrm{G}$ mobile networks. A thorough investigation of the mmWave system operation in a realistic urban environment is done in this paper. We presented two scenarios, such as, simulations with ray tracer and simulations with the use of ray tracer. We proposed an algorithm for mapping which is provided in the next section.

\section{Proposed method}

\subsection{System model}

The system model comprises of four major components, such as, scenario, ray-based modeler, system-level simulator, and performance evaluation, as shown in Fig. 3. Scenario illustrates the interaction with the proposed system, while ray-based model provides the real values. The 3D map and AP locations are provided to the ray-based modeler. Ray tracer give information about SINR and SNR to the SLS with the help of replacement algorithm. SLS runs the whole network simulation and provide the performance parameters for the evaluation. 


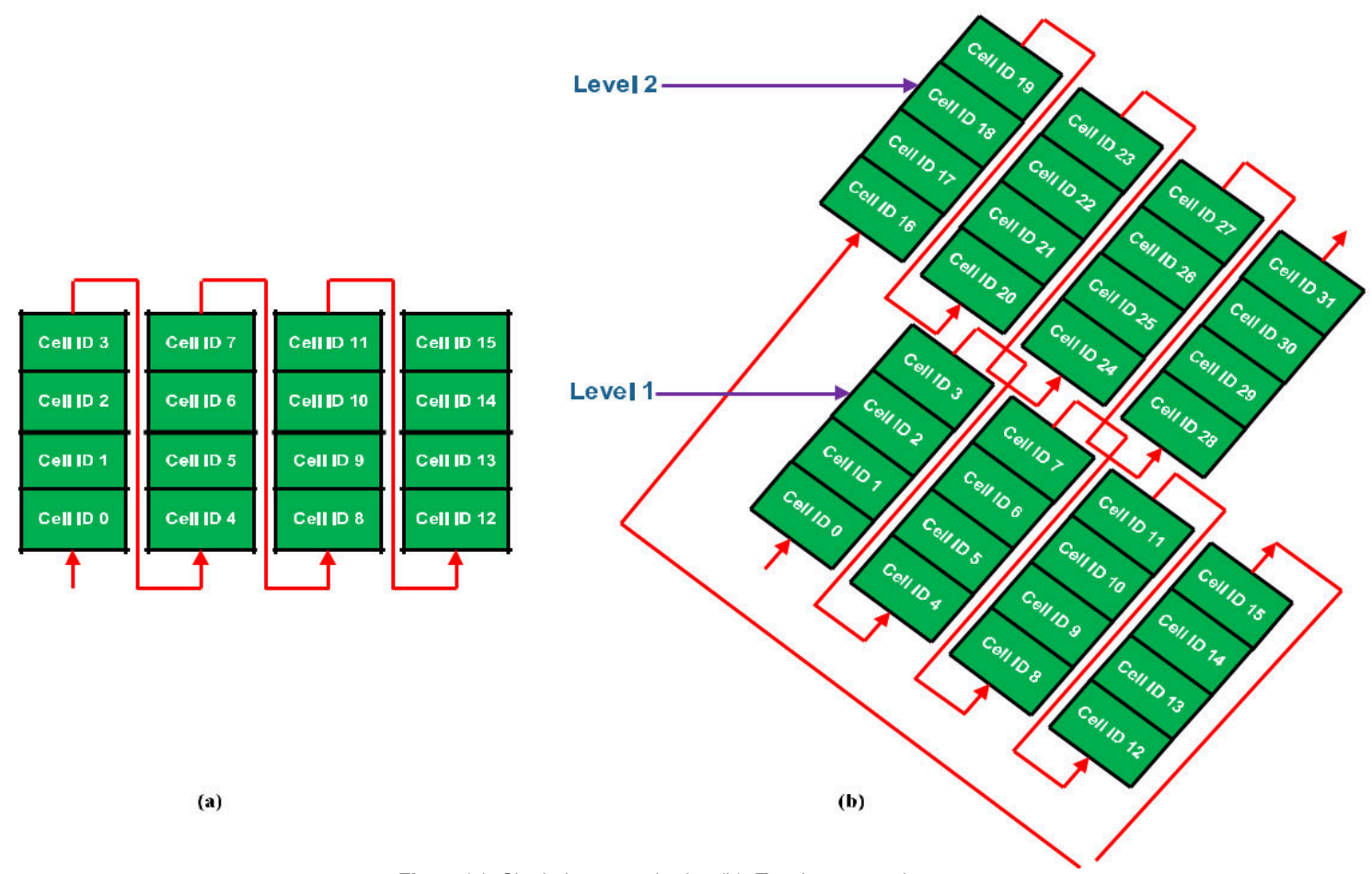

Fig. 1. (a). Single layer numbering (b). Two layers receiver area.

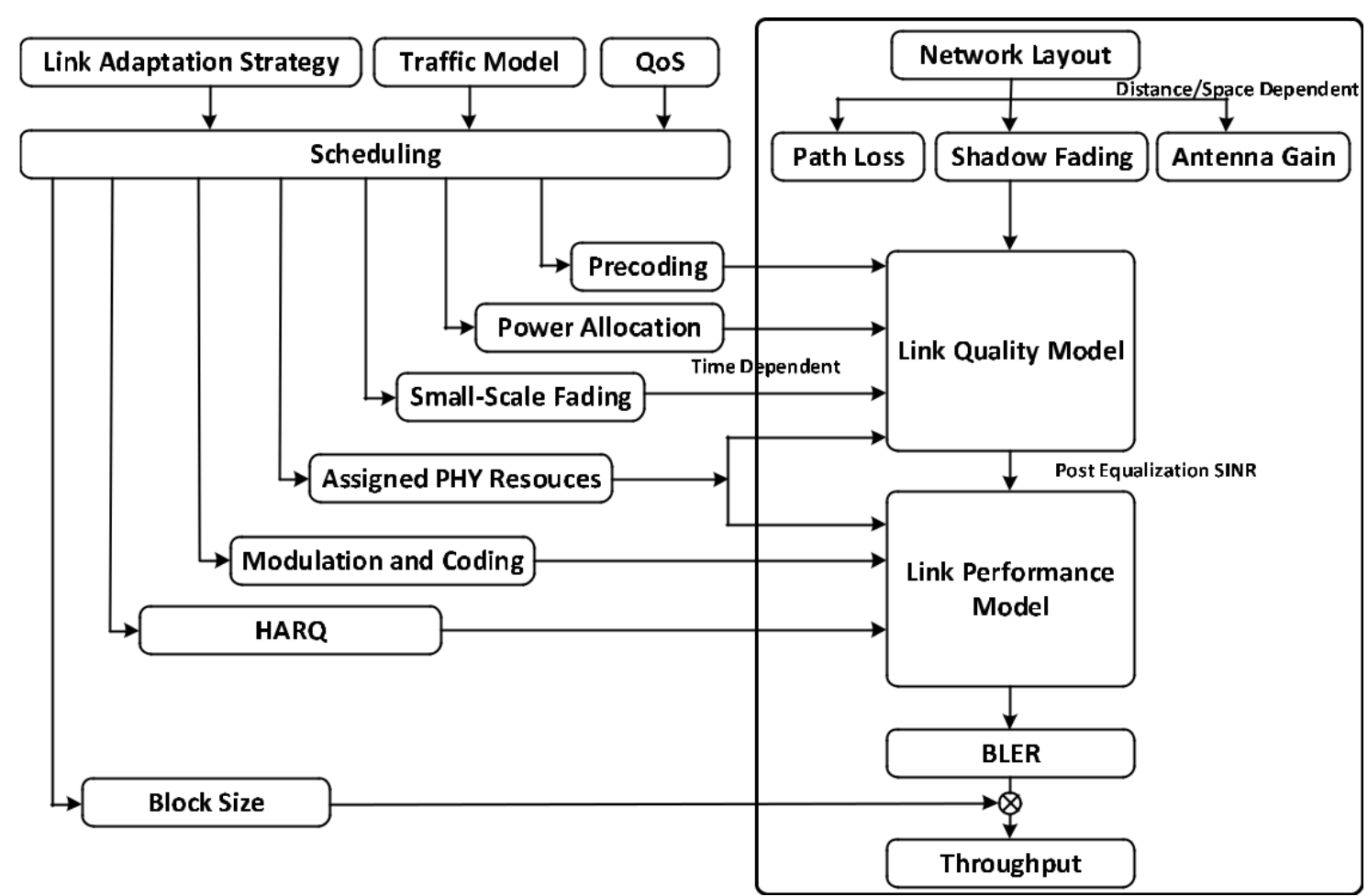

Fig. 2. Architecture of system-level simulator.

\subsection{Algorithm and mapping function}

The observational area is divided in 1 meter resolution provided from ray tracer and a definite deterministic value of ray tracer is injected to the SLS if the coordinates matches. The ray tracer provides deterministic rays for different layers with a particular layout which provides much better results. The mapping function picks the values from a particular coordinated location and compares with the values of predicted SLS ray values and if the values of ray tracer are better, then the values of the SLS are replaced with the ray tracer values. This ensures that a more accurate values for the nodes under consideration are used for the evaluation of system performance.

The mapping algorithm compares the location of each UE in ray tracer and SLS one by one. The flowchart is described in Fig. 4 . If the 
Table 1

Capabilities and limitations of ray tracer and SLS

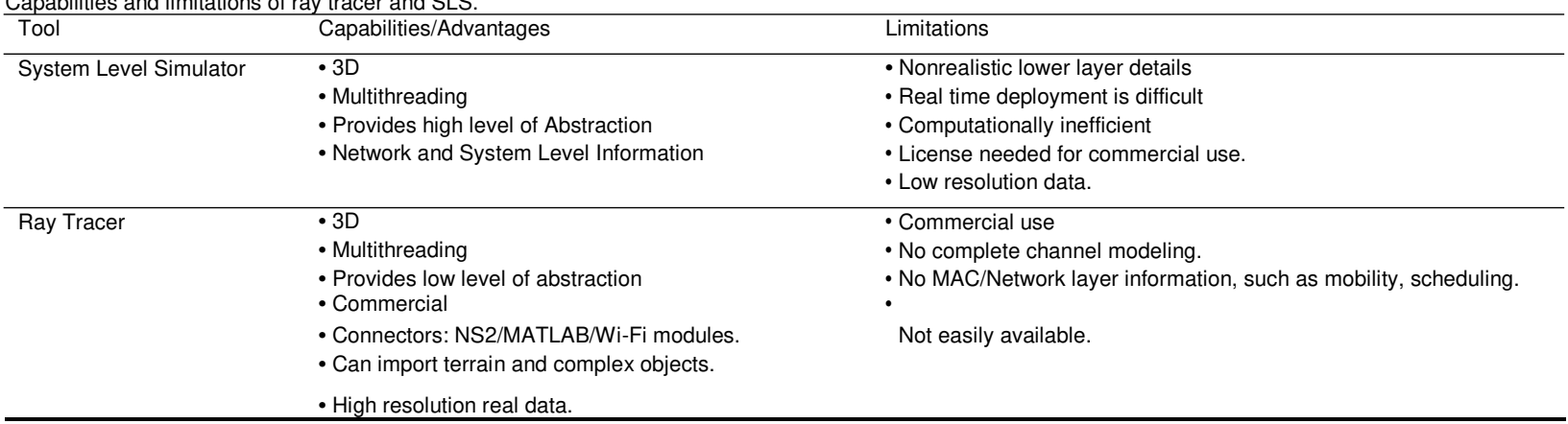

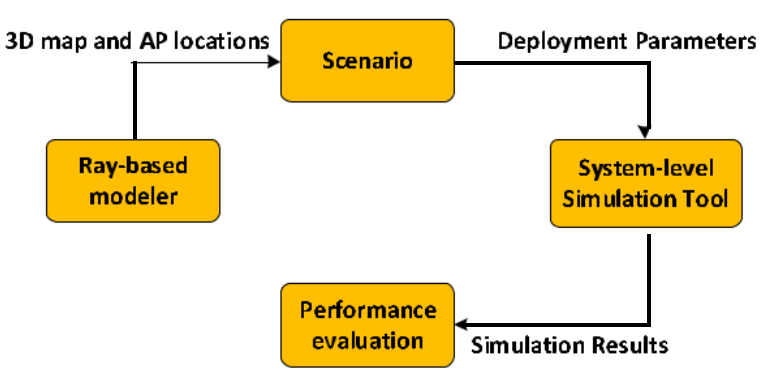

Fig. 3. System Model.

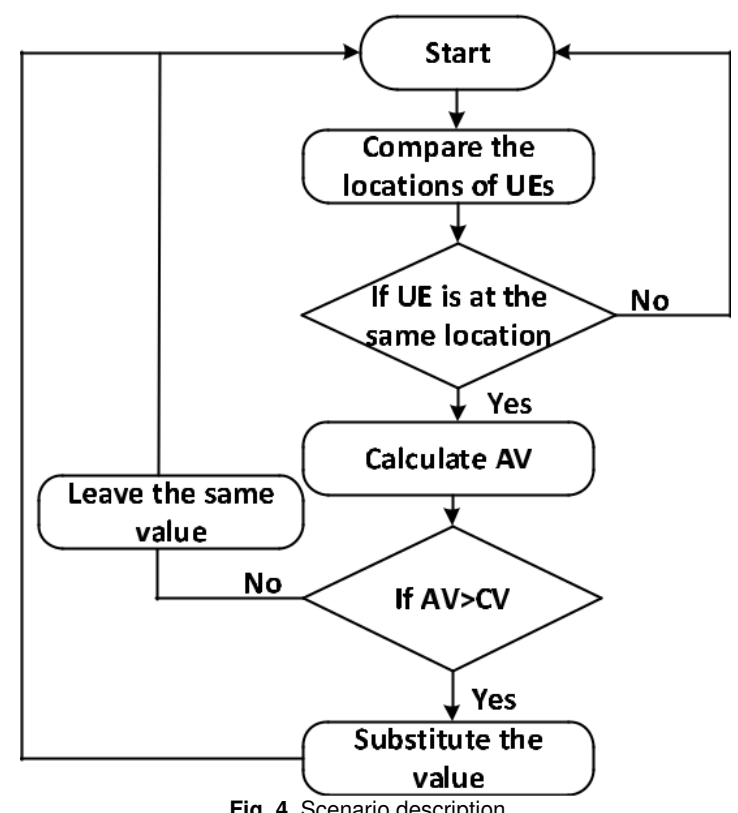

Fig. 4. Scenario description.

locations are same in both simulators, then an average of the values are calculated for ray tracer for values in the same region. If the average value $(\mathrm{AV})$ is greater than the current value $(\mathrm{CV})$ present in SLS, then the new value is substituted in the SLS entry, otherwise the values are unchanged. If the location is not the same, then the values next value in the ray tracer is picked. It keeps repeating the process until all the values from the ray tracer are mapped to the values needed in the SLS. Once the values are replaced from ray tracer to the SLS, the results are calculated for the whole system.
Table 2

Simulation parameters.

\begin{tabular}{ll}
\hline Parameter & Value \\
\hline Bandwidth & $20 \mathrm{MHz}$ \\
Carrier frequency & $2.6 \mathrm{GHz}$ \\
Noise power spectral density & $-174 \mathrm{dBm} / \mathrm{Hz}$ \\
Number of UEs & 20 \\
UE Speed & $5 \mathrm{~km} / \mathrm{h}(1.38 \mathrm{~m} / \mathrm{s})$ \\
Tx height & $25 \mathrm{~m}$ \\
Rx height & $1.5 \mathrm{~m}$ \\
Tx power & $46 \mathrm{dBm}$ \\
Number of runs & 100 \\
Simulation time & $1000 \mathrm{TTI}$ \\
\hline
\end{tabular}

\section{Simulations and results discussion}

\subsection{Simulation setup}

The simulation model consists of one transmitted eNodeB, $\mathrm{N}$ receiver User Equipment (UEs). In our experimentation, we use a singleinput single-output (SISO) system with constant distribution of 20 UEs in the cell. The distribution of the UEs are constant in each cell. The Tx height is 25 meters and UE with 1.5 meters on average. The UE mobility is taken as a walking person in urban scenario. The length of the simulation in Transmission Time Intervals (TTIs) are 1000. The simulation scenario parameters are summarized in Table 2. For the calculation of the distance, we used the two-point distance formula.

There are two scenarios used in this paper. In Scenario 1, the SNR values from the ray tracer are not fed into the SLS, and in Scenario 2 , the values of the ray tracer are used for the simulation. In our work, the scenarios included urban cells with a distance of 500 meters and with a UE mobility of $5 \mathrm{~km} / \mathrm{h}$ as an average pedestrian speed.

There are different parameters analyzed from the simulations, for example, considering the number of cells (eNodeBs), number of UEs pertaining to these eNodeBs, length of simulation in TTls, type of scheduler, average throughput values of UEs, average cell throughput, etc.

\subsection{Performance analysis}

In this work, we evaluated the performance of the system under the two considered scenarios using the following three metrics: average throughput, average spectral efficiency and average resource block (RB)/TTI/UE, as shown in Fig. 5. The values are presented in the following graph.

As shown in Fig. 5, in R1, a maximum of $2.82 \mathrm{Mb} / \mathrm{s}$ and a minimum of $0.09 \mathrm{Mb} / \mathrm{s}$ for UE 5 and UE 10 are observed, respectively. In R2, a maximum of $3.4 \mathrm{Mb} / \mathrm{s}$ for UE 2 and 4 , and a minimum of $0.42 \mathrm{Mb} / \mathrm{s}$ for UE 13 are observed. A mean value of all the UE's average throughput of $2.1 \mathrm{Mb} / \mathrm{s}$ is obtained in R1 and $2.116 \mathrm{Mb} / \mathrm{s}$ is obtained in R2.

For the comparison of spectral efficiency, individual values are calculated for all the UEs. In R3, the average spectral efficiency has a 


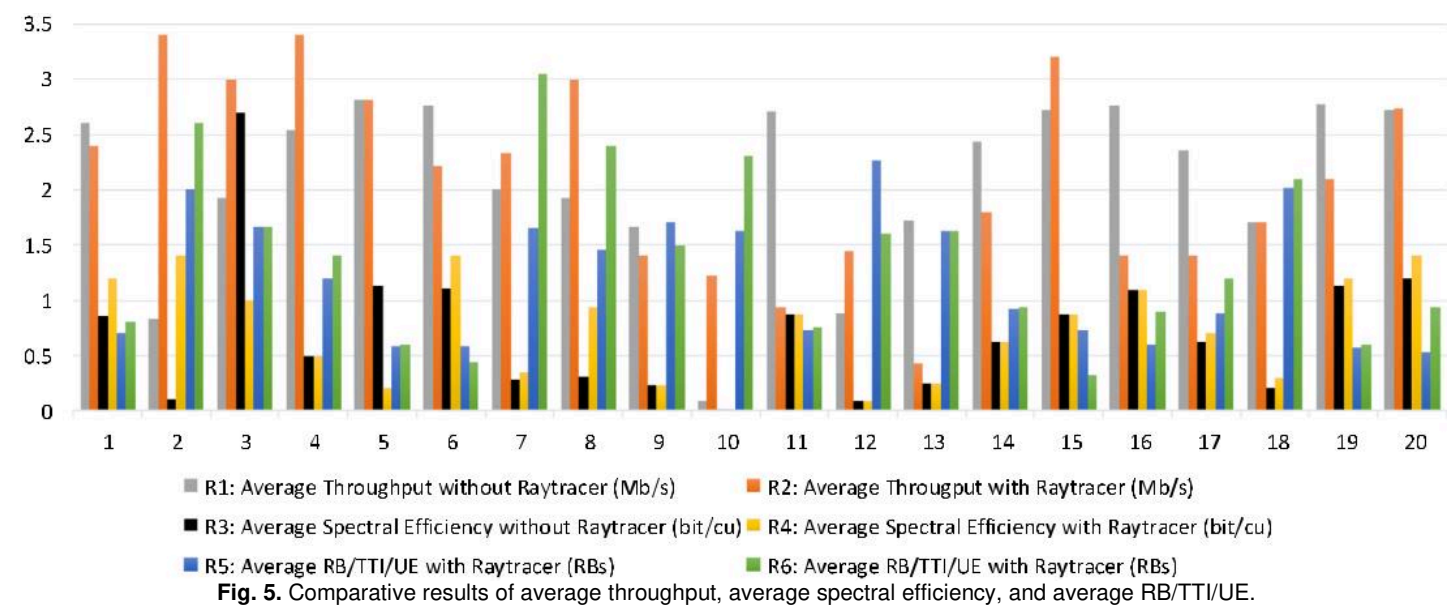

maximum value of $2.7 \mathrm{bit} / \mathrm{cu}$ for UE 3 and a minimum of $0.01 \mathrm{bit} / \mathrm{cu}$ for UE 10, respectively. For R4, a maximum value of $1.4 \mathrm{bit} / \mathrm{cu}$ of average spectral efficiency is observed for UE 2 and UE 20, and a minimum of $0.01 \mathrm{bit} / \mathrm{cu}$ for UE 10 is noted. The mean values for all the UEs remained $0.59 \mathrm{bit} / \mathrm{cu}$ for $\mathrm{R} 3$ and $0.73 \mathrm{bit} / \mathrm{cu}$ for $\mathrm{R} 4$, respectively.

The resource blocks per TTI per UE is calculated for both scenarios. In R5, $2.27 \mathrm{RB} / \mathrm{TTI} / \mathrm{UE}$ are allocated for UE 12 and a minimum of 0.58 $\mathrm{RB} / \mathrm{TTI} / \mathrm{UE}$ for UE 6. Similarly, in R6, a maximum value of $3.05 \mathrm{RB} / \mathrm{TTI} / \mathrm{UE}$ is observed for UE 7 and a minimum of $0.44 \mathrm{RB} / \mathrm{TTI} / \mathrm{UE}$ is observed for UE 6 , respectively. The mean value for all the UE's average RB/TTI/UE is 1.2 $\mathrm{RBs}$ for R5 and $1.386 \mathrm{RB} / \mathrm{TTI} / \mathrm{UE}$ for R6, respectively.

When simulating the $5 \mathrm{G}$ networks, this ray tracing mapping algorithm is a much better choice, though it is harder to get the images with ray tracer at different city maps, since the deployment of $5 \mathrm{G}$ setup is very limited. The proposed method is efficient and provides better results by replacing high resolution data through the help of ray tracer. In principle, this is very simple and easy to implement for future generation of wireless systems.

\section{Conclusions and future research directions}

Realistic channel modeling facilitates the system level simulations in enhancing the performance of wireless systems. In this paper, we obtained realistic values of channel from a ray tracer and proposed a novel mapping technique that maps the values of ray tracer to the system level simulator. We simulated the network in a MATLAB based system level simulator and results from our mapping techniques are compared with system that is not using ray tracing values. The results from our proposed mapping technique showed superiority in terms of average spectral efficiency and average throughput. The results showed that the proposed method has higher average throughput and average spectral efficiency. The proposed model can be used for other MIMO systems for better understanding of the system and implementation of the whole network. The work can be extended to multiple layers of the ray tracer values and research work could be used for dense network simulations. The current research work can be improved to provide third-party software with advanced functionalities, e.g., advanced data analysis. Different add-on features like cache management and resource management in a complex and dense simulations could be improved in the future versions of the simulator.

\section{Declaration of competing interest}

No author associated with this paper has disclosed any potential or pertinent conflicts which may be perceived to have impending conflict with this work. For full disclosure statements refer to https://doi.org/ 10.1016/j.comcom.2019.11.039.

\section{Acknowledgments}

This work is supported in part by the European Regional Development Fund (FEDER), through the Competitiveness and Internationalization Operational Programme (COMPETE 2020), Regional Operational Program of the Algarve (2020), Fundação para a ciên-cia e Tecnologia; i-Five: Extensão do acesso de espectro dinâmico para rádio 5G, POCI-01-0145-FEDER-030500 and in part by FCT/MEC through national funds under the project (THz-BEGUN under CMU Portugal call), CMU/ECE/0013/2017. The authors S. A. Busari and K. M. S. Huq also acknowledge the FCT for Ph.D. Grants under Reference PD/BD/113823/2015 and Postdoctoral Grants under Reference SFRH/BPD/110104/2015, respectively.

\section{References}

[1] J. Lota, S. Sun, T.S. Rappaport, A. Demosthenous, $5 \mathrm{~g}$ uniform linear arrays with beamforming and spatial multiplexing at $28,37,64$, and $71 \mathrm{GHz}$ for outdoor Urban communication: A two-level approach, IEEE Trans. Veh. Technol. 66 (11) (2017) 9972-9985.

[2] H. Chen, L. Liu, T. Novlan, J.D. Matyjas, B.L. Ng, J. Zhang, Spatial spectrum sensing-based device-to-device cellular networks, IEEE Trans. Wireless Commun. 15 (11) (2016) 7299-7313.

[3] M. Rupp, S. Schwarz, M. Taranetz, The vienna LTE-advanced simulators: Up and downlink, link and system level simulation, first ed. ser., in: Signals and Communication Technology, Springer, Singapore, 2016.

[4] M.K. Müller, et al., Flexible multi-node simulation of cellular mobile communications: the vienna 5G system level simulator, J. Wirel. Commun. Netw. (2018) $1-17$

[5] J.C. Ikuno, M. Wrulich, M. Rupp, System level simulation of LTE networks, in: 2010 IEEE 71st Vehicular Technology Conference, Taipei, 2010, pp. 1-5.

[6] J. Olsson, J. Asante, 5G Simulation Framework, Dissertation, Department of Electrical Engineering, Linköping University, Sweden, 2018.

[7] C. Jao, et al., WiSE: A system-level simulator for $5 \mathrm{G}$ mobile networks, IEEE Wirel. Commun. 25 (2) (2018) 4-7.

[8] S. Pratschner, M. Klaus Muller, F. Ademaj, A. Nabavi, B. Tahir, S. Schwarz, M. Ruppy, Verification of the Vienna 5G Link and System Level Simulators and their Interaction, 2019, pp. 1-8.

[9] B. Ai, et al., On indoor millimeter wave massive MIMO channels: Measurement and simulation, IEEE J. Sel. Areas Commun. 35 (7) (2017) 1678-1690.

[10] C. Teh, B. Chung, E. Lim, An accurate and efficient 3-D shooting-and- bouncingpolygon ray tracer for radio propagation modeling, IEEE Trans. Antennas and Propagation 66 (12) (2018) 7244-7254.

[11] R.E. Chall, B. Miscopein, D. Kténas, Unii-mac protocol: Design and evaluation for $5 \mathrm{G}$ ultra-dense small cell networks operating in $5 \mathrm{GHz}$ unlicensed spectrum, Comput. Commun. 126 (2018) 11-27.

[12] M. Celaya-Echarri, L. Azpilicueta, P. Lopez-Iturri, E. Aguirre, S. De MiguelBilbao, V. Ramos, F. Falcone, Spatial characterization of personal rf-emf exposure in public transportation buses, IEEE Access 7 (2019) 33 038-33 054.

[13] S. Mumtaz, L.T. Tu, K. Saidul, A. Gameiro, CaLibration and evaluation of fixed and mobile relay-based system level simulator, J. Comput. Syst. Netw. Commun. 2014 (2010) http://dx.doi.org/10.1155/2010/149257, Article ID 149257, 14 pages.

14] F. Hossain, T.K. Geok, T.A. Rahman, M.N. Hindia, K. Dimyati, S. Ahmed, C.P. Tso, N.Z. Abd Rahman, An efficient 3-D ray tracing method: prediction of indoor radio propagation at $28 \mathrm{GHz}$ in $5 \mathrm{G}$ network, Electronics 8 (2019) 286.

[15] Sigint, Trunet wireless \& fractal networx LTD, 2019, [Online] Available: http: //www.fractalnetworx.com/. (Accessed: 18-Oct-2019). 
2019-12-02

\section{A novel mapping technique for ray tracer to system-level simulation}

Awais Khan, Muhammad

Elsevier

Awais Khan M, Adeshina Busari S, Mohammed Saidul Huq K, et al., (2020) A novel mapping technique for ray tracer to system-level simulation. Computer Communications, Volume 150, January 2020, pp. 378-383

https://doi.org/10.1016/j.comcom.2019.11.039

Downloaded from Cranfield Library Services E-Repository 